\title{
Asthma-associated variants induce IL33 differential expression through a novel regulatory region
}

Ivy Aneas $^{1 * \wedge}$, Donna C. Decker ${ }^{2 *}$, Chanie L. Howard², Débora R. Sobreira1, Noboru J. Sakabe ${ }^{1}$, Kelly M. Blaine ${ }^{2}$, Michelle M. Stein ${ }^{1}$, Cara L. Hrusch², Lindsey E. Montefiori ${ }^{1}$, Juan Tena ${ }^{3}$, Kevin M. Magnaye ${ }^{1}$, Selene M. Clay ${ }^{1}$ James E. Gern ${ }^{4}$, Daniel J. Jackson ${ }^{4}$, Matthew C. Altman ${ }^{5}$, Edward T. Naureckas ${ }^{2}$, Douglas K. Hogarth ${ }^{2}$, Steven R. White ${ }^{2}$, 8 Jose Luis Gomez-Skarmeta ${ }^{3}$, Nathan Schoetler ${ }^{1,2}$, Carole Ober ${ }^{1}$, Anne I. Sperling ${ }^{2 \wedge}$,

9 Marcelo A. Nobrega ${ }^{1 \wedge}$,

11 Authors affiliations:

12 1- Department of Human Genetics, University of Chicago, Chicago, IL 60637, USA.

13 2- Department of Medicine, Section of Pulmonary and Critical Care Medicine, University of

14 Chicago, Chicago, IL 60637, USA.

15 3- Centro Andaluz de Biología del Desarrollo (CSIC/UPO/JA), Universidad Pablo de Olavide,

16 Seville 41013, Spain.

17 4- Department of Pediatrics, University of Wisconsin School of Medicine and Public Health,

18 Madison, WI 53726, USA

19 5- Division of Allergy and Infectious Diseases, Department of Medicine, University of

20 Washington, Seattle, WA 98195, USA

21 *These authors contributed equally to this study

${ }^{\wedge}$ Corresponding Authors:

24 Anne I. Sperling

25 Section of Pulmonary and Critical Care, Dept of Medicine

26 University of Chicago

27924 E. 57th Street

28 Chicago, IL 60637

29 asperlin@uchicago.edu

31 Marcelo A. Nobrega

32 Department of Human Genetics

33 University of Chicago

34920 E 58 ${ }^{\text {th }}$ St CLSC 515-E

35 nobrega@uchicago.edu

Ivy Aneas

38 Department of Human Genetics

39 University of Chicago

40920 E 58 $8^{\text {th }}$ St CLSC 515-E

41 ianeas@bsd.uchicago.edu 


\section{ABSTRACT}

43 Genome-wide association studies (GWAS) have implicated the IL33 locus in asthma,

44 but the underlying mechanisms remain unclear. Here, we identify a $5 \mathrm{~kb}$ region within

45 the GWAS-defined segment that acts as a strong regulatory element in vivo and in vitro.

46 Chromatin conformation capture showed that this $5 \mathrm{~kb}$ region loops to the IL33

47 promoter, potentially regulating its expression. Supporting this notion, we show that

48 genotype at an asthma-associated SNP, rs1888909, located within the $5 \mathrm{~kb}$ region, is

49 associated with IL33 gene expression in human airway epithelial cells and IL-33 protein

50 expression in human plasma, potentially through differential binding of OCT-1

51 (POU2F1) to the asthma-risk allele. Our data demonstrate that asthma-associated

52 variants at the IL33 locus mediate allele-specific regulatory activity and IL33 expression,

53 providing a novel mechanism through which a regulatory SNP contributes to genetic risk

54 of asthma. 


\section{Introduction}

Asthma is a common and chronic inflammatory disease of the airways, with significant contributions from both genetic and environmental factors. Genetic factors account for more than half of the overall disease liability ${ }^{1}$ and GWAS have discovered $>60$ loci contributing to asthma disease risk $^{2}$, with most of the associated variants

61 located in noncoding regions. Linking these noncoding variants to genes and

62 understanding the mechanisms through which they impart disease risk remains an

63 outstanding task for nearly all asthma GWAS loci.

Among the most highly replicated asthma loci are variants near the genes encoding the cytokine IL-33 on chromosome 9p24.1 and its receptor, ST2 (encoded by IL 1RL1), on chromosome $2 q 12.1$, highlighting the potential importance of this pathway in the genetic etiology of asthma. A crucial role for IL-33 in allergic airway inflammation and bronchial airway hyperresponsiveness has been known since its discovery in $2005^{3}$. Studies in individuals with asthma and in murine asthma models have identified elevated levels of IL-33 protein in both sera and tissues ${ }^{4,5}$. This cytokine is a potent

71 inducer of type 2 immune responses through its receptor ST2, and has been broadly

72 implicated in other allergic and inflammatory conditions, such as atopic dermatitis,

73 allergic rhinitis, and eosinophilic esophagitis $6,7,8$.

75 risk at the IL33 GWAS locus reside within a linkage disequilibrium (LD) block in a

76 noncoding genomic segment located $2.3 \mathrm{~kb}$ upstream of th $/ L 33$ gene. We therefore

77 posited that variants in this region impact on IL33 expression by altering cis-regulatory

78 element(s) that control quantitative, spatial and/or temporal-specific gene expression. 
79 Previous studies of complex diseases have shown how regulatory variants in promoters

80 and enhancer elements lead to an increased risk of disease through altering the

81 expression of nearby genes $9,10,11,12,13$. In contrast, other types of cis-regulatory

82 elements, including repressors and insulators (also known as enhancer blocking

83 elements), are less understood and characterized than enhancers, but are also likely to

84 be functionally modified by regulatory variants ${ }^{14}$.

Here, we combined genetic fine-mapping using GWAS data sets combined with

86 functional annotations from relevant tissues to dissect the asthma-associated region

87 upstream of the IL33 gene. We identified a regulatory element harboring SNPs that control IL33 expression. Genotypes at rs1888909, a SNP within this regulatory element,

89 are associated with IL33 expression in ethnically diverse populations, as well as IL-33

90 plasma protein levels. Our study provides functional insights into the role of common

91 regulatory variants at the IL33 locus and illustrate how a causal SNP can exert

92 phenotypic effects by modulating the function of regulatory elements that do not fit into

93 standard definitions of enhancers, insulators, or repressors.

96 Results

97 Defining the IL33 locus asthma-associated critical region

Variants at the IL33 locus have been robustly associated with asthma in GWAS

99 of ethnically-diverse populations ${ }^{2}, 15,16,17,18,19,20$. We first used LD between the most

100 significantly associated SNP in each GWAS (referred to here on as the lead SNP) and

101 other SNPs to define the region harboring potentially causal variants at this locus (Fig. 
102 1a). Five lead SNPs were reported among seven large GWAS, defining an LD block

103 spanning 41 kb in European ancestry individuals (chr9: 6,172,380-6,213,468, hg19;

104 Supplementary Fig. 1a), which included 21 additional SNPs in LD $\left(r^{2} \geq 0.80\right)$ with at

105 least one of the five lead SNPs (26 total SNPs). Because LD tracts are longer in

106 European genomes compared to African ancestry genomes, we also sought results of

107 GWAS in African Americans to potentially narrow this region. Three multi-ancestry

108 GWAS 16, 18, 19 included African Americans, but only one ${ }^{16}$ provided GWAS results

109 separately by ancestry. In that study, the lead GWAS SNP rs1888909 differed from the

110 lead SNPs in the European ancestry (rs1342326 ${ }^{15}, \mathrm{rs} 928413^{17}, \mathrm{rs} 7848215^{20}$ and

111 rs992969²) or combined multi-ancestry (rs2381416 ${ }^{16}$ and rs992969 ${ }^{18,19}$ ) GWAS.

112 Because there was so little LD in this region in African Americans, we used an $r^{2} \geq 0.40$

113 to define LD. SNPs in LD with rs1888909 at $r^{2} \geq 0.4$ in African Americans defined a

114 region of 20 kb (chr9: 6,188,124-6,209,099, hg19; Supplementary Fig. 1b and

115 Supplementary Table 1) that excluded two lead SNPs (rs928413 ${ }^{17}$ and rs7848215 ${ }^{20}$ ).

116 To gain more formal statistical support for this interval, we next used a Bayesian

117 method, Sum of Single Effects (SuSiE) ${ }^{21}$, to fine-map the associated region and identify

118 credible sets (CS) of SNPs at the IL33 locus with high probabilities of being causal.

119 Within each CS, variants are assigned posterior inclusion probabilities (PIPs), with

120 higher PIPs reflecting higher probabilities of being causal and the sum of all PIPs within

121 a CS always equaling 1. Using all SNPs at this locus from a GWAS of childhood onset

122 asthma ${ }^{2}$ in British white subjects from the UK Biobank ${ }^{22}$, we identified three CSs

123 (Fig.1b). CS1 contained variants with the highest PIPs and included six SNPs, all

124 among those defined by LD with the lead GWAS SNPS, defining a $12.7 \mathrm{~kb}$ region 
125 approximately $22 \mathrm{~kb}$ upstream of the transcriptional start site of the IL33 gene (chr9:

126 6,197,392-6,210,099; hg19) and overlapping with the regions defined by LD (Fig. 1a

127 and Supplementary Table 2). The three CS1 SNPs with the highest PIPs included the

128 lead SNPs in two multi-ancestry GWAS (rs992969; PIP 0.391), the lead SNP in the

129 African American GWAS ${ }^{16}$ (rs1888909; PIP 0.209), and a SNP in LD with the lead

130 SNPs (rs3939286; PIP 0.205). We note that two other CSs of 25 SNPs (CS2) and 28

131 SNPs (CS3) were identified by SuSiE, suggesting additional, independent regions

132 potentially regulating the expression of IL33 or other genes.

Next, we used Roadmap Epigenome ${ }^{23}$ and $\mathrm{ENCODE}^{24}$ data to annotate the

134 regulatory landscape of the more inclusive $20 \mathrm{~kb}$ asthma-associated interval defined by

135 LD. This segment was enriched with chromatin marks and DNase hypersensitive sites

136 (Fig. 1C), suggestive of regulatory potential in multiple cell types. We also identified two

137 CTCF sites within $2 \mathrm{~kb}$ of each other with evidence of CTCF binding in multiple cell lines

138 (84 cell lines have CTCF binding to site 1 and 142 have CTCF binding to site 2 out 194

139 ENCODE-3 lines). In addition, the cohesin complex RAD21-SMC-3 subunits and zinc-

140 finger proteins such as ZNF384 and ZNF143 also bind to this region (Fig. 1d). Binding

141 of this multi-subunit complex along with CTCF can provide sequence specificity for

142 chromatin looping to promoters or have insulator functions ${ }^{25,} 26$.

143 Interestingly, the three lead GWAS SNPs ( $r s 1342326^{15}$, rs $2381416^{16}$ and

144 rs9929692, 18, 19) in the LD-defined $20 \mathrm{~kb}$ region did not overlap with regions of open

145 chromatin or transcription factor binding; one, rs992969², 18, 19, mapped within

146 heterochromatin (thick blue horizontal bar) in virtually every ENCODE cell line

147 (Supplementary Fig. 2). Heterochromatin is highly compacted region in the genome and 
148 not actively involved in gene regulation. For these reasons, it is not likely any of these

149 three lead SNPs within the LD-defined region are causative of the asthma association.

150 In contrast, five SNPs in high LD with the asthma-associated lead SNPs, including two

151 from CS1 and including the lead African American GWAS SNP (rs1888909), overlapped

152 with a region of open chromatin and CTCF, cohesin and ZNF binding, delineating a

153 discrete 5 kb region (chr9: 6,194,500-6,199,500, hg19) (Fig. 1c-d). Collectively, these

154 data reveal a $5 \mathrm{~kb}$ region that harbors both asthma-associated SNPs and marks of

155 regulatory activity that could modulate IL33 expression through long-range interactions. 
a

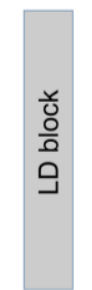

b

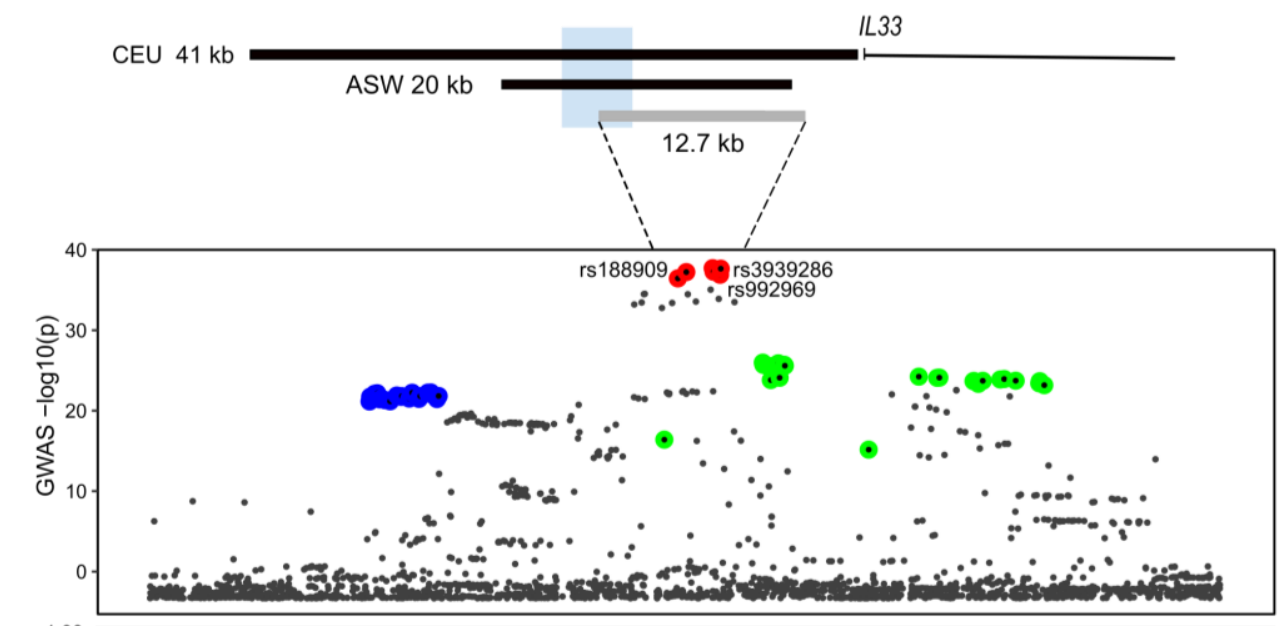

W
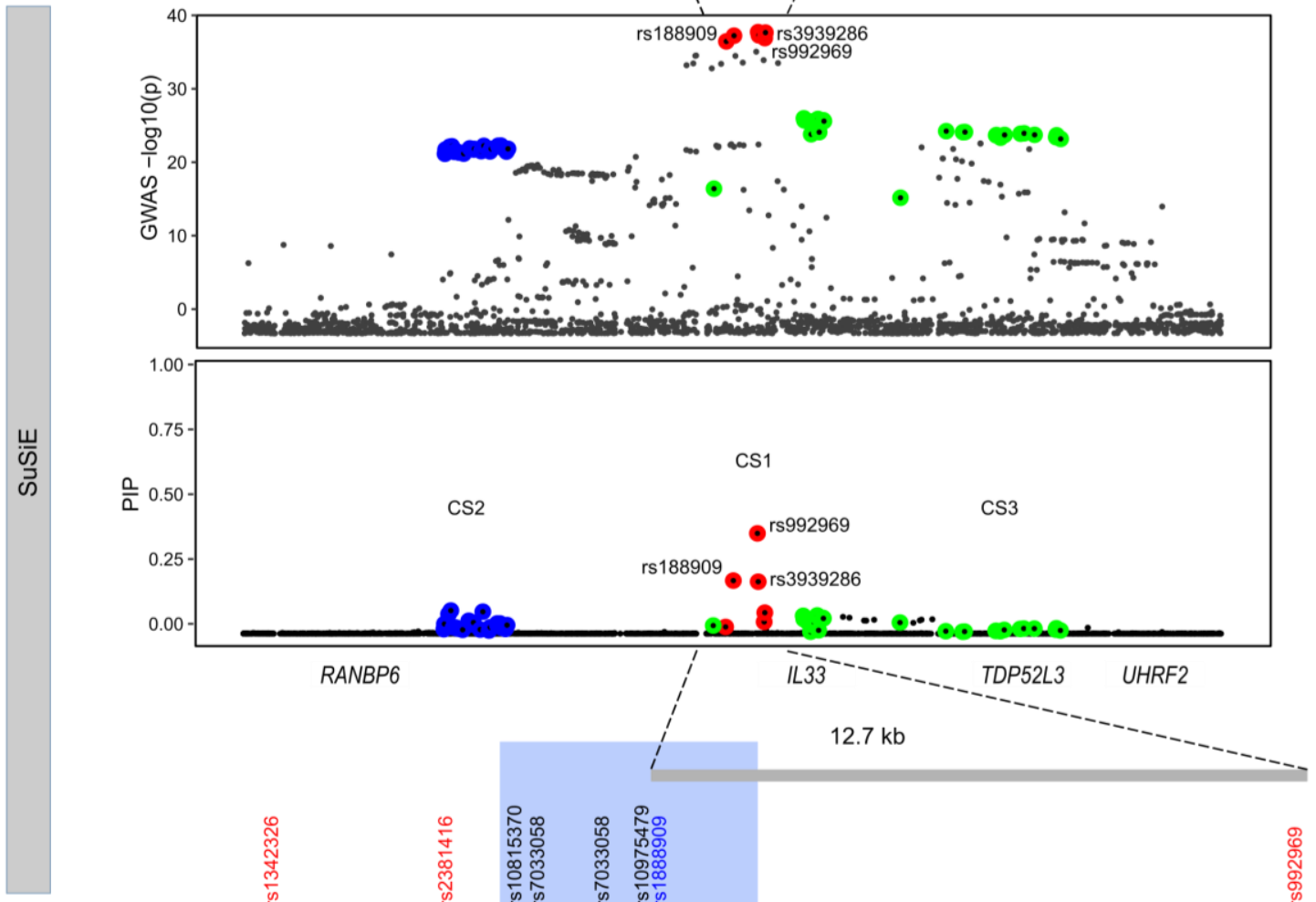

C

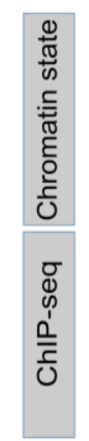

ASW $20 \mathrm{~kb}$

DHS

Figure 1. Epigenetic characterization of the asthma-associated critical region in the IL33 locus. a

158 Schematic organization of the IL33 gene and the asthma-associated region (black bars) of European

159 ancestry (CEU 41 kb, chr9: 6,172,380-6,213,468; hg19) and African ancestry (ASW 20 kb, chr9:

$1606,188,124-6,209,099 ;$ hg19) positioned upstream of exon 1. b Significance of SNP association in the

161 GWAS $^{2}$ (top) and fine mapping results (bottom) for each variant at the IL33 locus (bottom). Colors 
162 indicate each credible set (CS) identified. CS1: red, CS2 blue; CS3 green. CS1 (rs992969, rs1888909, 163 rs3939286) defines a region of $12.7 \mathrm{~kb}$ (chr9: 6,197,392 6,210,099; hg19). c Position of the lead GWAS

164 SNPs (in red) and additional SNPs in high LD ( $\left.r^{2} \geq 0.8\right)$ with the lead SNPs (in black) within the ASW $20 \mathrm{~kb}$

165 LD region. The lead SNP rs1888909 in African ancestry is shown in blue. Chromatin states from

166 Roadmap Epigenomics Project showing regions with potential regulatory activity. Yellow: active

167 enhancer; green: transcribed sequence; blue: heterochromatin. DNase hypersensitive (DHS) sites

168 indicating open chromatin regions are shown. Tissues (from the top): E096 Lung primary HMM; E095 Left

169 ventricle primary HMM; E116 GM128781 Lymphoblastoid cell primary HMM, E122 HUVEC Umbilical Vein

170 Endothelial Primary Cells Primary HMM d ChIP-seq data from ENCODE-3 cell lines (338 factors; 130 cell

171 types) showing co-binding of CTCF, RAD2, ZFNs and SMC-3 at the $5 \mathrm{~kb}$ interval (blue shaded region;

172 chr9: 6,194,500-6,199,500; hg19).

173

174

175 Regulatory properties of a $5 \mathbf{k b}$ region upstream of IL33

Having identified a region of interest, we sought to determine its impact on IL33

177 expression. Because IL33 is expressed in multiple tissues, we used an in vivo model

178 system to assay for the spatial regulatory properties of this $5 \mathrm{~kb}$ region. However, the

179 lack of evolutionary conservation at the locus between human and mouse

180 (Supplementary Fig. 3) required the creation of a "humanized" transgenic mouse model.

181 For this, we used a human Bacterial Artificial Chromosome (BAC - clone RP11-

$182725 \mathrm{~F} 15)$, approximately $166 \mathrm{~kb}$ long, spanning the coding region of the IL33 gene and

183 its upstream sequences, including the $20 \mathrm{~kb}$ asthma-associated region. We recombined

184 a cassette containing a E2-Crimson reporter with a 3' stop codon into exon 2, in frame

185 with the IL33 start codon (ATG). Any IL33 regulatory regions within this BAC would

186 drive E2-Crimson expression, mimicking IL33 endogenous spatio-temporal expression

187 patterns. Also, to directly test the regulatory impact of the $5 \mathrm{~kb}$ region, we selectively

188 deleted this asthma-associated DNA segment from the full BAC and assessed the 
resultant IL33 expression in vivo, in mice harboring the full length or the $5 \mathrm{~kb}$ deletion

190 BACs (Fig. 2a).

192 mice ( $\left.\mathrm{h} / \mathrm{L} 33^{\text {Crim }} \mathrm{BAC}\right)$ showed that E2-Crimson fluorescent protein is highly expressed in

193 this tissue (Fig. 2b, upper panel and Supplementary Fig. 4a). Strikingly, constitutive

194 expression of the E2-Crimson reporter was co-localized with the endothelial marker

195 CD31 and observed in high endothelial venule (HEV) cells in mouse lymph nodes. This

196 observation validates the species-specificity of the E2-Crimson expression, as previous

197 studies report that while IL-33 is produced by HEV in humans, it is not found in murine

$198 \mathrm{HEV}^{\mathbf{2 7}}$. In contrast, deletion of the $5 \mathrm{~kb}$ region in the reporter BAC ( $\mathrm{h} / L 33^{\text {CrimBAC5kdel) }}$

199 significantly depleted E2-Crimson immunostaining in lymph nodes compared to full BAC

200 mice (Fig. 2b, lower panel and Supplementary Fig. 4b). The 5 kb deletion also

201 significantly reduced E2-Crimson mRNA expression in heart and lung in 3 out of 4

202 independent lines (Fig. 2c). These results show that the BAC encodes human-specific

203 regulatory patterns in vivo and demonstrate the importance of the $5 \mathrm{~kb}$ noncoding

204 segment for proper IL33 expression. 
a

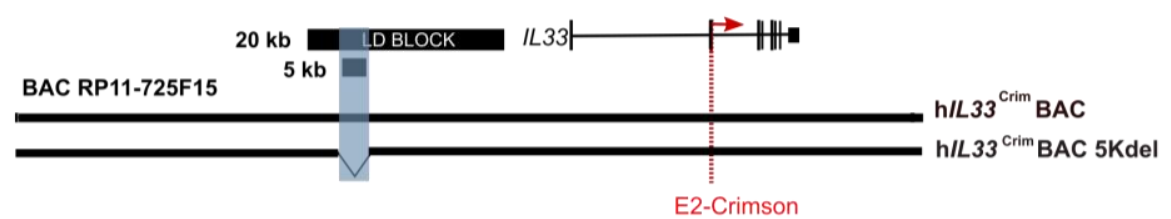

b
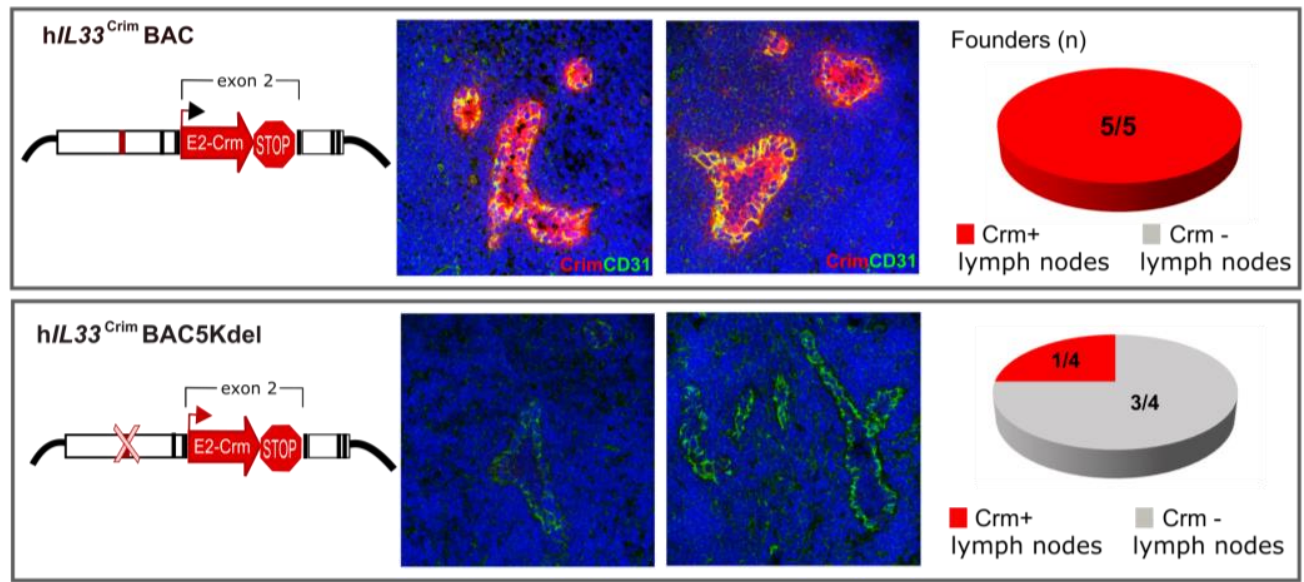

c

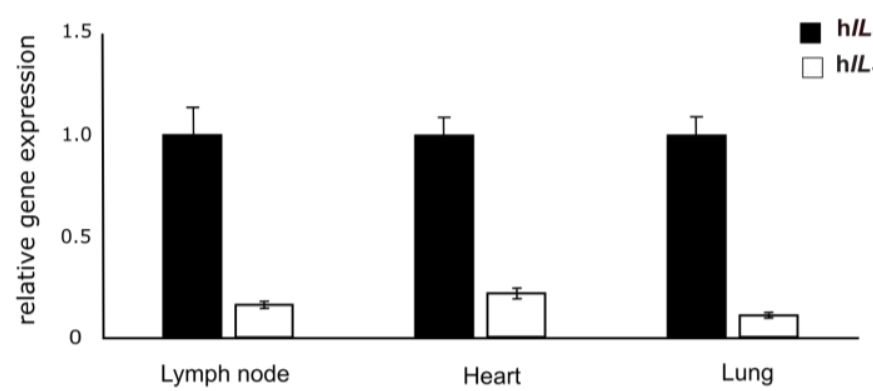

211

212 Figure 2. The IL33-containing BAC in transgenic mice encodes human-specific regulatory patterns

213 and demonstrates the importance of the $\mathbf{5} \mathbf{~ k b}$ noncoding segment for proper IL33 expression. a

214 Schematic of human BAC clone RP11-725F15 (166 kb) spanning the entire coding region of IL33 and its

215 upstream region including the $20 \mathrm{~kb}$ asthma-associated interval and the $5 \mathrm{~kb}$ region of interest shaded in

216 blue (black bars). To produce a human IL33 reporter strain, a cassette containing E2-Crimson with a stop

217 sequence was inserted into exon 2, in frame with the IL33 translational start site (red dotted line).

218 Transgenic mice were generated with either the full BAC (hIL33CrimBAC) or a BAC containing a deletion of

219 the $5 \mathrm{~kb}$ interval within the LD block (h/L33Crim BAC5Kdel). b Immunofluorescence staining of mouse

220 peripheral lymph node sections of E2-Crimson in $\mathrm{h} / L 33^{\text {Crim }} \mathrm{BAC}$ mice (upper panels) or $\mathrm{h} / L 33^{\text {Crim }}$

221 BAC5Kdel (lower panels). Representative founder BAC transgenic mice are shown. Sections were

222 stained with anti-E2-Crimson (red) and the mouse endothelial cell marker CD31 (green). Hoescht staining

223 for nuclei is in blue. c qPCR analysis of E2-Crimson mRNA obtained from lymph node (LN), heart and

224 lung in both BAC strains are shown 


\section{Long-range chromatin interactions in the IL33 locus}

226

227

228

229

230

231

232

233

234

235

236

237

238

239

240

241

242

243

244

245

246

247

We next assessed the physical interactions between the asthma-associated region and the IL33 promoters, by performing circular chromosome conformation capture followed by high-throughput sequencing (4C-seq) in cells obtained from

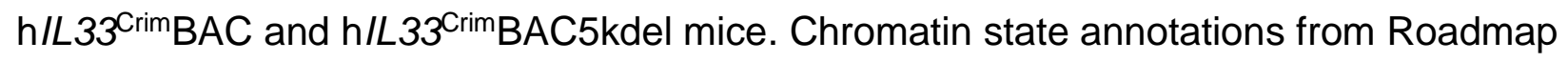
Epigenome suggest the presence of distal regulatory elements, such as enhancers (yellow bars) distributed across the human BAC region containing the $I L 33$ gene and its upstream non-coding DNA (Fig. 3a).

We positioned the viewpoint directly upstream of the $5 \mathrm{~kb}$ region in order to capture interactions between this genomic region and the entire adjacent IL33 locus

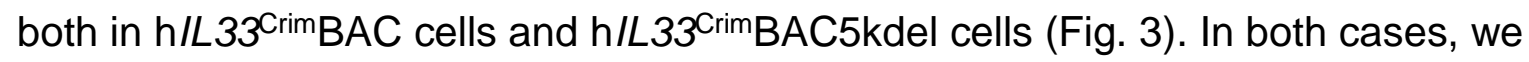
observed interactions between the viewpoint and several potential regulatory regions located upstream and downstream (gray arcs), including the less frequently used promoter of the long transcript as well as the predominantly used promoter for the short transcript of IL33 (blue arcs). Deletion of the $5 \mathrm{~kb}$ fragment reduced the interactions at the IL33 locus compared to those observed using the full BAC, including the loss of the interaction with the short transcript promoter, confirming the necessity of this region for proper IL33 regulation and explaining the loss of E2-Crimson expression in h/L33 CrimBAC5kdel mice (Fig. 2b-c).

Because we are limited to measure interactions within the $166 \mathrm{~kb}$ of the human $\mathrm{BAC}$ in mouse cells, we cannot exclude the possibility that the $5 \mathrm{~kb}$ region also physically interacts with and regulates other genes in the topological associated domain (TAD) containing IL33 in humans. To address this possibility, we carried out promoter 
248 capture Hi-C in human lymphoblastoid cell lines (LCLs). Our data show interactions

249 between the asthma-associated LD block region and both IL33 promoters but not to

250 other genes (Supplementary Fig.5). These data further support the notion that the

251 asthma-associated LD region harbors regulatory elements that physically interact with

252 IL33 to regulate its expression.

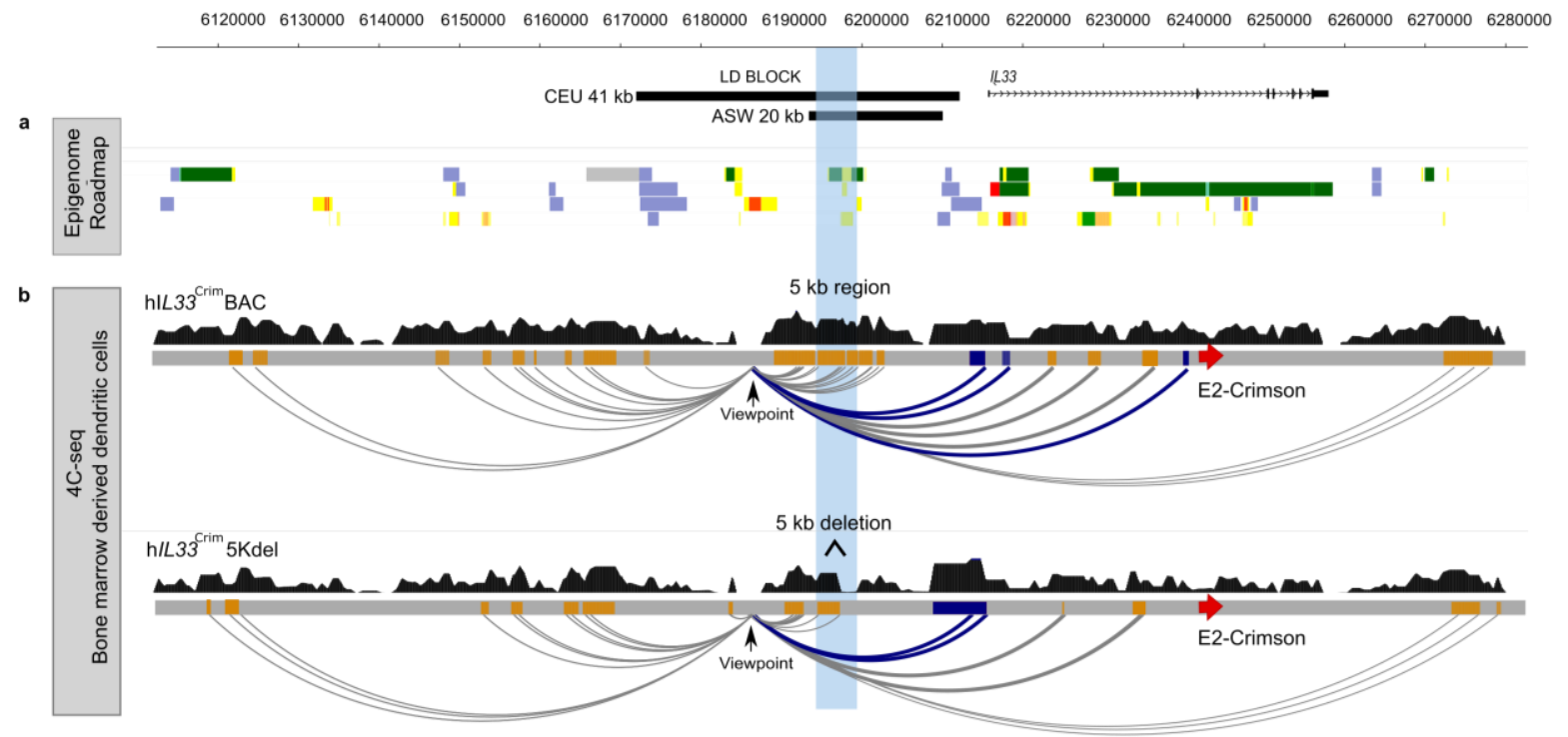

Figure 3. Interaction maps show looping of the asthma-associated region to the IL33 promoter. a

255 Roadmap Epigenome data from heart (E095 Left ventricle primary HMM), lung (E096 Lung primary

256 HMM), LCL (E116 GM128781 Lymphoblastoid cell primary HMM) and endothelial cells (E122 HUVEC

257 Umbilical Vein Endothelial Primary Cells Primary HM). Chromatin state assignments are indicated as:

258 transcribed, green; active enhancer, yellow; active promoter, red; repressed, gray; heterochromatin, blue.

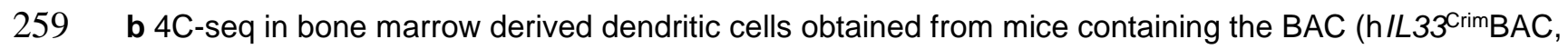

260 top) and a deletion of the $5 \mathrm{~kb}$ interval within the LD block (h/L33Crim BAC5Kdel, bottom). Gray bars

261 correspond to a schematic representation of the human BAC showing the E2-Crimson reporter insertion

262 site on exon 2 of the IL33 gene (red arrow) and the asthma associated $5 \mathrm{~kb}$ region (blue shaded box). In

263 both experiments, reads were mapped to the coordinates corresponding to the human BAC (chr9:

$2646,112,733-6,279,294$; hg19) and peaks shown in dark blue and orange represent the promoter and distal

265 elements, respectively. Arcs depict interactions from the viewpoint located upstream the $5 \mathrm{~kb}$ region.

266 Interactions between the LD region, near to the $5 \mathrm{~kb}$ region (viewpoint), and the IL33 promoters are noted 267 in blue 
Asthma-associated SNPs modify regulatory properties of the $5 \mathrm{~kb}$ region

To functionally characterize the regulatory impact of allelic variants of asthmaassociated SNPs in the $5 \mathrm{~kb}$ region, we utilized a combination of in vitro and in vivo

271 reporter assays. Because this region overlaps with chromatin states suggestive of

272 enhancer function in some cell types, and because its deletion in the BAC resulted in a

273 generalized loss of reporter expression in vivo, we hypothesized that the $5 \mathrm{~kb}$ region

274 corresponds to an enhancer. To test the regulatory potential of this DNA segment in

275 vitro, we cloned both the risk and non-risk haplotypes of the $5 \mathrm{~kb}$ fragment, as well as a

276 shorter $1 \mathrm{~kb}$ fragment centered on the accessible chromatin segment from ENCODE

277 (Fig. 1c) in a luciferase vector and transfected them in human cell lines. We used an

278 immortalized human aortic endothelial cell line (TeloHAEC), as the ENCODE project

279 chromatin data annotate this region as a putative enhancer in endothelial cells. We

280 failed to detect enhancer activity of either haplotype in either the $5 \mathrm{~kb}$ or $1 \mathrm{~kb}$ fragments

281 (Supplementary Fig. 6).

Interestingly, upon close inspection of ENCODE data we notice that this $5 \mathrm{~kb}$

283 region is not bound by transcription factors usually associated with enhancer activity,

284 including transcriptional activators, repressors, or RNA Poll. Rather, it is bound by both

285 CTCF and subunits of the cohesin complex across multiple cell lines (Fig. 1d). Both

286 CTCF and cohesin are key determinants of chromatin loop formation and stabilization,

287 including the positioning of regulatory elements close to the promoters of their target

288 genes, or serving as insulators, with enhancer blocking properties.

To test the enhancer blocking properties of the $5 \mathrm{~kb}$ fragment, we first used an in

290 vitro luciferase-based assay in which candidate sequences are cloned between a strong 
291 promoter (SV40) and a strong enhancer (HS2 element in human beta-globin LCR) ${ }^{28}$.

292 Luciferase expression is driven by the enhancer and promoter elements, and a

293 decrease in luciferase activity would be interpreted as enhancer blocking activity, with

294 the enhancer not able to loop to the adjacent SV40 promoter. When the $5 \mathrm{~kb}$ region was

295 cloned into this vector we observed prominent enhancer-blocking activity (Fig. 4a) and

296 significant differences were also observed between fragments containing either the risk

297 or non-risk alleles for the five SNPs, with the risk allele showing weakened enhancer

298 blocking activity (Fig. 4b; $p=0.001$ ). As a control, we used a DNA sequence located 60

$299 \mathrm{~kb}$ upstream of IL33 and devoid of any epigenetic marks of active chromatin and with no

300 evidence of CTCF or cohesin binding. This control sequence had no significant impact

301 on the reporter gene expression (Fig. 4a).

302

To test the this enhancer blocking property in vivo we used a zebrafish reporter

303 assay $^{29}$. The $5 \mathrm{~kb}$ region was cloned in a reporter cassette containing Green

304 Fluorescent Protein (GFP) driven by a cardiac actin promoter and a midbrain enhancer.

305 An enhancer blocking element cloned in this vector would restrict the access of the

306 midbrain enhancer to the GFP reporter gene, while the ability of the actin promoter to

307 activate GFP in skeletal muscle and somites would be maintained (Fig. 4c). In these

308 experiments, the $5 \mathrm{~kb}$ region led to a decreased mid-brain-specific GFP signal when

309 compared to the control sequence, which displayed no enhancer blocking activity (Fig.

$3104 d ; p<0.001)$. Similar to the data in the in vitro luciferase experiments, there was a

311 significant difference in GFP activity between fragments containing the risk or non-risk

312 alleles $(p=0.041)$, with the risk alleles resulting in reduced enhancer blocking activity.

313 Taken together, our data provide evidence that the $5 \mathrm{~kb}$ region has enhancer blocking 
314 properties in vitro and in vivo, and that alleles of asthma-associated SNPs within this

315 region are able to modulate this property.

317 asthma-associated SNPs. To determine the impact of the SNPs on its regulatory

318 function, we cloned a smaller $1 \mathrm{~kb}$ fragment, including one CTCF site and the SNPS

319 rs10975479 and rs1888909, located 15 bp apart from each other, and displaying the

320 highest LD $\left(\mathrm{r}^{2}\right)$ with the tag SNP rs992969 (0.56 and0.96, in CEU respectively). This

321 segment of the $5 \mathrm{~kb}$ region overlaps with the minimal critical region predicted by fine-

322 mapping by SuSie (Fig. 1a). This shorter fragment still showed prominent enhancer

323 blocking activity in vitro, in luciferase assays (Fig. 4e). Furthermore, deletion of a 400 bp

324 within the $1 \mathrm{~kb}$ element, harboring the SNPs rs10975479 and rs1888909, abrogated the

325 enhancer blocking activity of this fragment. Importantly, this 400 bp deletion does not

326 span the CTCF binding site within the $1 \mathrm{~kb}$ fragment. Testing these fragments in our in

327 vivo zebrafish transgenic reporter assay confirmed the $1 \mathrm{~kb}$ fragment enhancer blocking

328 activity and the functional importance of one or both SNPs within the 400 bp deleted

329 region in regulating GFP expression (Fig. 4f). Together, these data characterize a

330 regulatory region upstream of the IL33 locus and implicate the asthma-associated SNPS

331 rs10975479 and rs1888909 in regulating IL33 expression. 
a

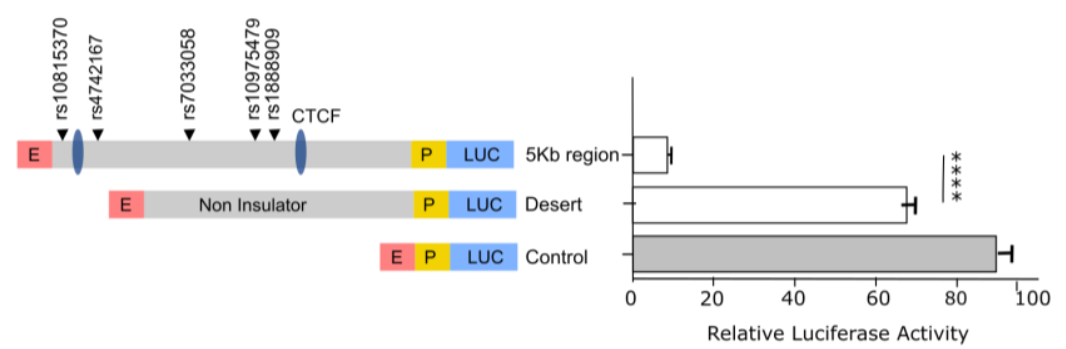

b

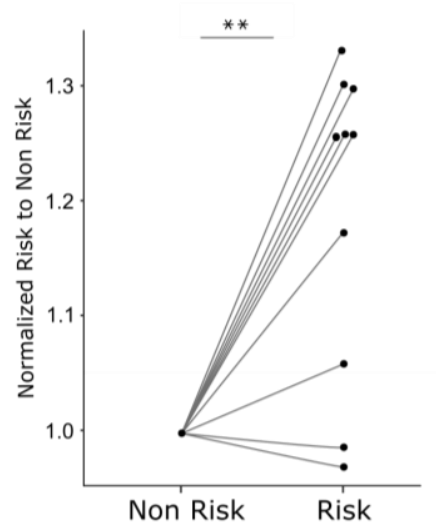

d

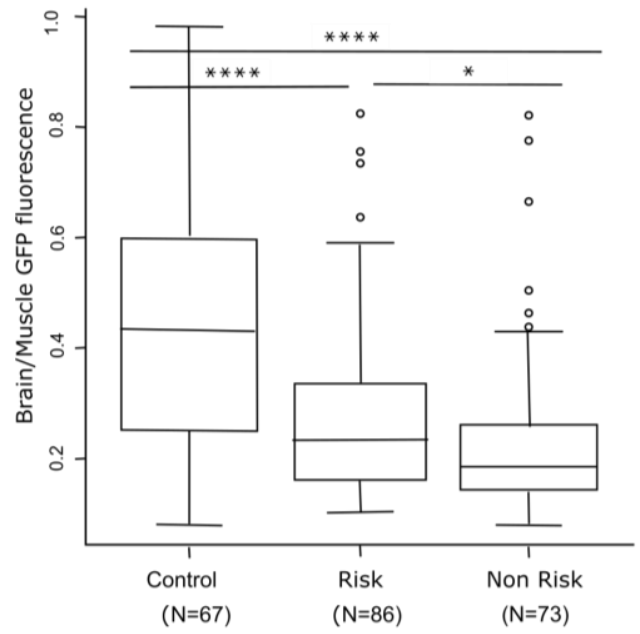

e
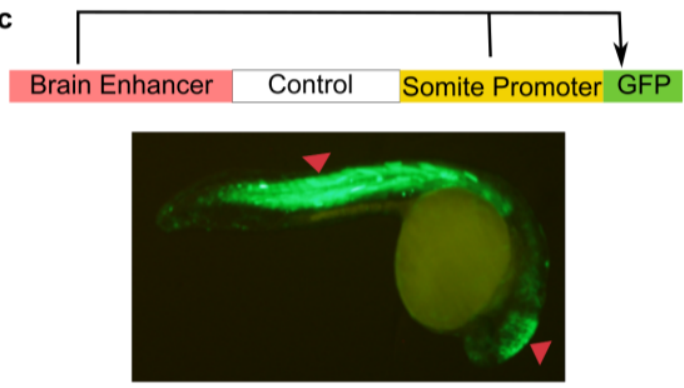

Brain Enhancer $5 \mathrm{~kb}$ region Somite Promoter GFP
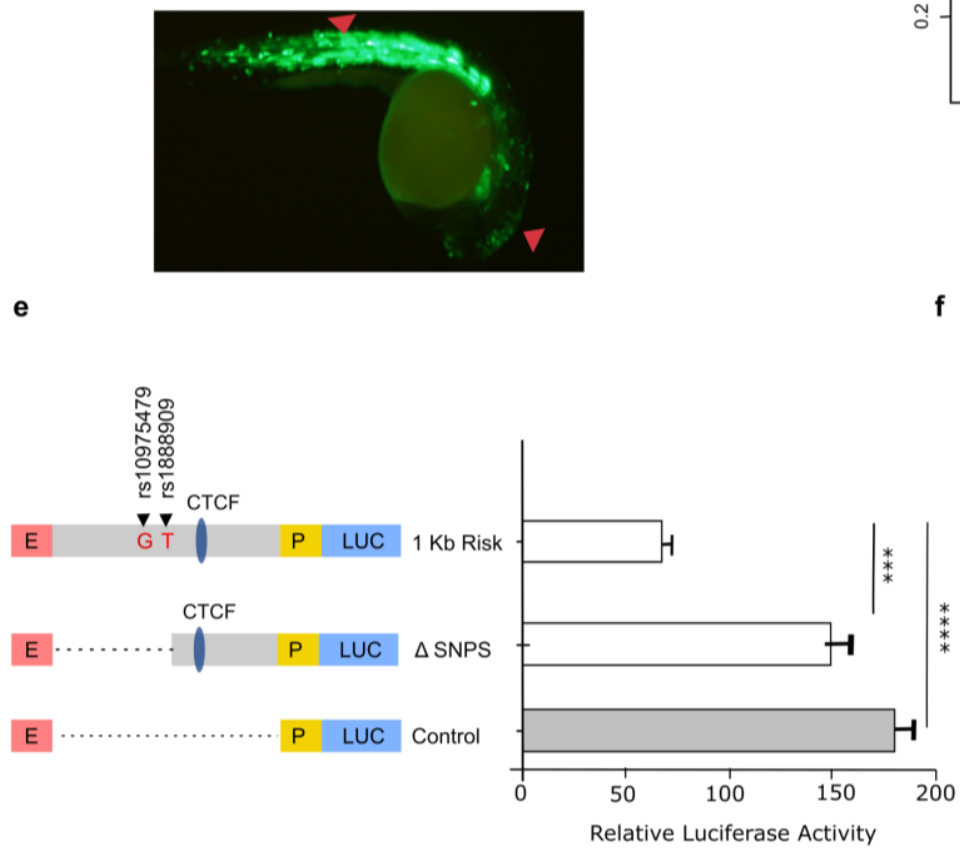

334 Figure 4. Impact of the asthma-associated variants in the regulatory property of the $\mathbf{5} \mathbf{k b}$ region. a

335 In vitro transgenic reporter assay. Luciferase based enhancer barrier assay using $5 \mathrm{~kb}$ constructs (chr9: 
6,194,500-6,199,500; hg19) that were cloned between HS2 enhancer (E) and SV40 promoter (P) sequences. SNPs in the construct are noted (black arrowheads). Results are from 3 independent experiments. ${ }^{* * *} \mathrm{p}<0.0001$, unpaired t-test. $\mathbf{b}$ Luciferase activity values of the risk construct is shown as fold change over the activity obtained in the non-risk sequence. Results shown represent data from 10 independent experiments. ${ }^{* *} \mathrm{p}=0.0014$, two-way ANOVA. c In vivo zebrafish transgenic reporter assay.

341 Green fluorescent protein (GFP) expression 24 hours post fertilization (hpf) in mosaic F0 embryos

342 injected with vectors containing a control sequence (top panel) or $5 \mathrm{~kb}$ interval sequence (bottom panel).

343 d Comparison between $5 \mathrm{~kb}$ constructs containing risk or non-risk alleles for enhancer blocking property.

344 Data is presented as midbrain/somites EGFP intensity ratio compared with empty gateway vector which 345 has no enhancer blocking activity. ${ }^{* * *} p=1.1 \mathrm{e}-5 ;{ }^{*} p=0.041$, ANOVA pairwise T-test. e $1 \mathrm{~kb}$ DNA fragment 346 (chr9: 6,197,000-6,197,917; hg19) containing the risk alleles for asthma variants rs10975479 and 347 rs1888909 or a 400bp deletion in the region harboring those SNPs (chr9: 6,197,399-6,197,914; hg19) 348 were assayed for luciferase assay in K562 cells. Results are representative of 3 independent experiments

$349{ }^{* * *} \mathrm{p}=0.0005$, unpaired t-test. $\mathrm{f}$ Zebrafish reporter assay comparing the $1 \mathrm{~kb}$ DNA fragment and fragment 350 with the $400 \mathrm{bp}$ deletion $(\triangle \mathrm{SNP})$. Data are presented as in (d).

\section{Differential binding of OCT-1 to allelic variants of rs1888909} hypothesized that the risk alleles of rs1888909 may alter transcription factor binding, resulting in altered regulatory activity and IL33 expression. Although not predicted to be a causal SNP (Fig. 1b), we also studied rs10975479 (G/A) as it is only 15 bp away from

357 rs1888909. We performed an electrophoretic mobility shift assay (EMSA) using small

358 labeled DNA probes and unlabeled competitors spanning four different combinations of

359 the risk or non-risk alleles for variants rs10975479 (G/A) and rs1888909 (T/C). Upon

360 incubation with nuclear extract, we observed differences in binding patterns between

361 the risk (G-T) and non-risk (A-C) probes, suggesting that different transcription factor 
362 binding complexes are formed in the presence of these two alleles (Fig. 5a). The major

363 changes in binding are observed with both probes carrying the rs1888909 (T) allele,

364 thereby indicating that the difference is driven by the risk allele T of this SNP. These

365 data demonstrate that risk allele (T) of the asthma-associated SNP rs1888909 alters

366 protein binding properties, possibly influencing IL33 expression.

To identify nuclear proteins that bind to the rs1888909 (T) vs rs1888909 (C)

368 probes, we isolated 3 bands from the non-risk rs1888909 (C) lane, and 2 bands from

369 the risk rs1888909 (T) lane for mass spectrometry analysis (Supplementary Fig. 7). As

370 a control, we isolated the same regions in the lane where the lysate had been incubated

371 with a cold probe competitor. After filtering out the proteins that were also found on the

372 control lane and the nuclear proteins that aren't known to bind to DNA, we identified 3

373 transcription factors bound only to the non-risk probe, NFE2, TFCP2 and FOXL2 and 4

374 transcription factors bound only to the risk probe, OCT-1 (POU2F1), FOXP1, STAT3

375 and STAT5b. We then used the UniPROBE protein array database ${ }^{31}$ to select the

376 transcription factors known to bind to at least one octamer containing a risk or non-risk

377 allele. This analysis resulted in one transcription factor: OCT-1 (POU2F1), which bound

378 to the EMSA probe and was differentially bound to the risk allele. OCT-1 was selected

379 as our primary candidate for further investigation.

380 To confirm OCT-1-specific binding to the risk probe we first used a cold

381 competition assay and demonstrated that the band specifically competed with an OCT-1

382 canonical DNA binding motif, but not with a mutated oligonucleotide (Fig. 5b, left panel).

383 Conversely, binding to the non-risk A-C probe was not competed by the consensus or

384 mutated OCT-1 oligonucleotides, demonstrating specificity for OCT-1 association with 
385 only the risk allele (Fig. 5b, right panel). Further, an OCT-1 specific antibody

386 supershifted the nuclear complex formed with the rs1888909 (T) probe (Fig. 5c). We

387 were able to visualize this shift independently of the order of incubation of the nuclear

388 extract with probe or antibody, suggesting robust protein binding. We then performed

389 chromatin immunoprecipitation (ChIP) followed by qPCR to demonstrate enrichment of

390 OCT-1 binding to the DNA region containing the rs18889099 (T) (Fig. 5d). These

391 experiments provide strong evidence that OCT-1 binds differentially to the risk allele

392 and directs the formation of differential allelic nuclear complexes at the rs1888909

393 variant. 


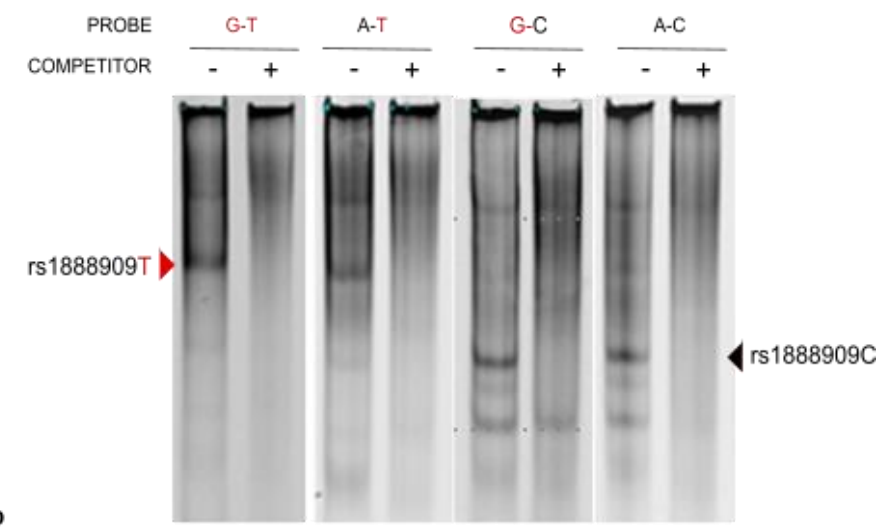

b
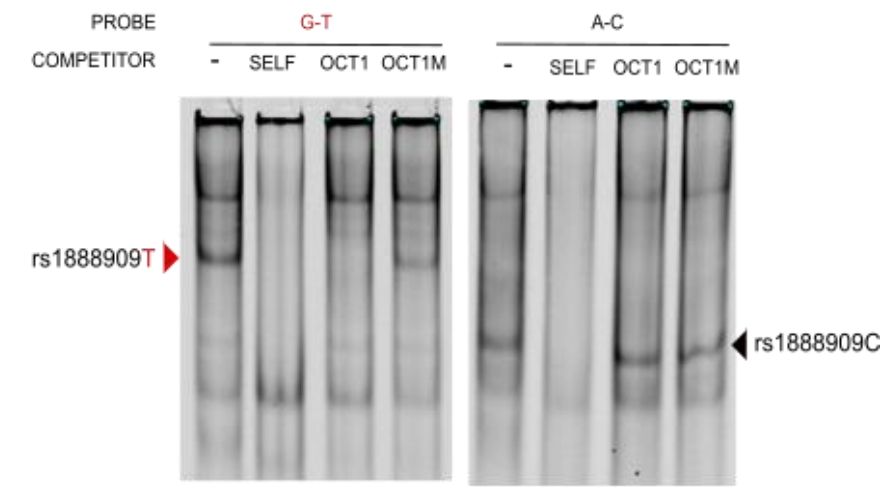

c

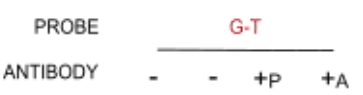

d
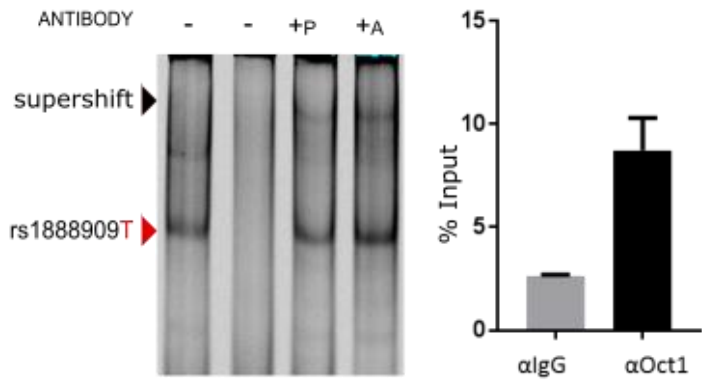

395 Figure 5. Regulatory region containing the risk allele rs1888909T selectively binds OCT-1.

396 a Radiolabeled probes carrying the risk (in red) and/or non-risk sequences for SNPs rs 10975479 and

397 rs1888909 were incubated with nuclear extract obtained from K562 cells. Different complexes formed by

398 rs1888909 are marked by red or black arrows. b Cold competition assay with OCT-1 consensus (OCT1)

399 or mutated OCT-1 (Oct1M) oligonucleotides. EMSA probes and oligo competitor (100x molar excess) are

400 noted above each gel. c Supershift complex formation with addition of anti-OCT-1 antibody as indicated

401 by the red arrow. $+\mathrm{P}$ indicates addition of probe with nuclear extract, followed by incubation with antibody.

$402+$ A indicates incubation of extract with antibody followed by addition of probe. $d$ Chromatin

403 immunoprecipitation of H292 chromatin with anti-OCT-1 antibody shows percent enrichment to input

404 chromatin compared to control lgG antibody. Results are representative of 3 independent experiments 


\section{Asthma-associated SNPs are associated with IL33 mRNA and IL-33 protein levels}

406

407

408

409

410

411

412

413

414

415

416

417

418

419

420

421

422

423

424

425

426

427

Finally, we tested the functional consequences of the GWAS SNPs rs1888909, rs10975479 and rs992969 on IL33 mRNA and IL-33 protein abundance. The first two SNPs are within the $5 \mathrm{~kb}$ region. The latter, rs992969, is located outside of this region, but was previously reported to be associated with IL33 expression in bronchial epithelial cells from primarily non-Hispanic white subjects ${ }^{32}$. SNP rs992969 is in high LD with rs1888909 in European ancestry populations ( $r^{2}=1$ in CEU), but less so in African American populations ( $r^{2}=0.45$ in ASW), and in low LD with rs 10975479 in both populations ( $r^{2}=0.56$ and 0.13 , respectively) (Supplementary Figure 1 and Supplementary Table 1). We used RNA-seq data from endobronchial brushings obtained from 123 asthmatic and non-asthmatic adult subjects, mostly of European ancestry (Fig. 6a), and from nasal epithelial cell brushings from 189 African American children from high risk asthma families (Fig. 6b). In both populations, carriers of one or two copies of the rs1888909 (T) risk allele had significantly higher IL33 transcript levels compared to non-carriers of this allele. Genotypes at rs992969 were more modestly associated with transcript levels and only significant in the African American samples after correcting for multiple (3) tests; rs10975479 was not associated with IL33 transcript abundance in either sample (Fig. 6a-b, Supplementary Table 3). These results are consistent with the EMSA data suggesting that allelic variants of rs1888909 result in differential protein binding. The stronger effects of rs1888909 on IL33 expression in both populations suggest that rs1888909 is the causal variant in this region and that associations with other variants (such as rs992969) in GWAS and in gene expression studies were due to LD with the causal variant. 
We observed similar patterns of association in studies of IL-33 protein levels in

429 plasma from 30 children $^{33}$ of European ancestry (Fig. 6b). Children who carried the

430 asthma risk alleles at rs1888909 or rs992969 had more IL-33 protein compared to

431 children not carrying these alleles. There was no association between genotype at SNP

432 rs10975479 and IL-33 protein levels.

433 The aggregate of our mouse transgenic assays, in vivo and in vitro reporter

434 assays, and EMSA collectively supported a role for rs1888909 as a causal variant for

435 the association with IL33 expression. The associations between the risk alleles and

436 increased IL33 transcript levels and IL-33 protein abundance further support a role for

437 this region in regulating the $I L 33$ gene and pointing to rs 1888909 as the causal variant

438 in this region, corroborating predictions from our in vitro and in vivo studies. 
a
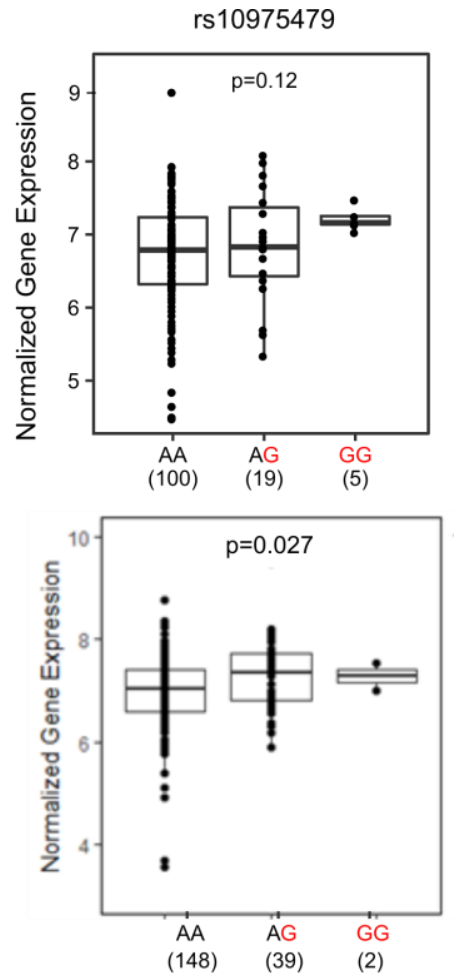

b

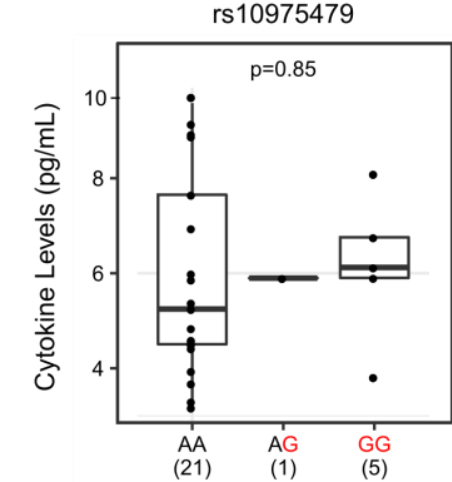

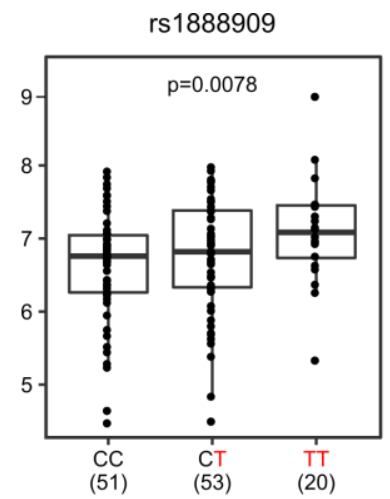
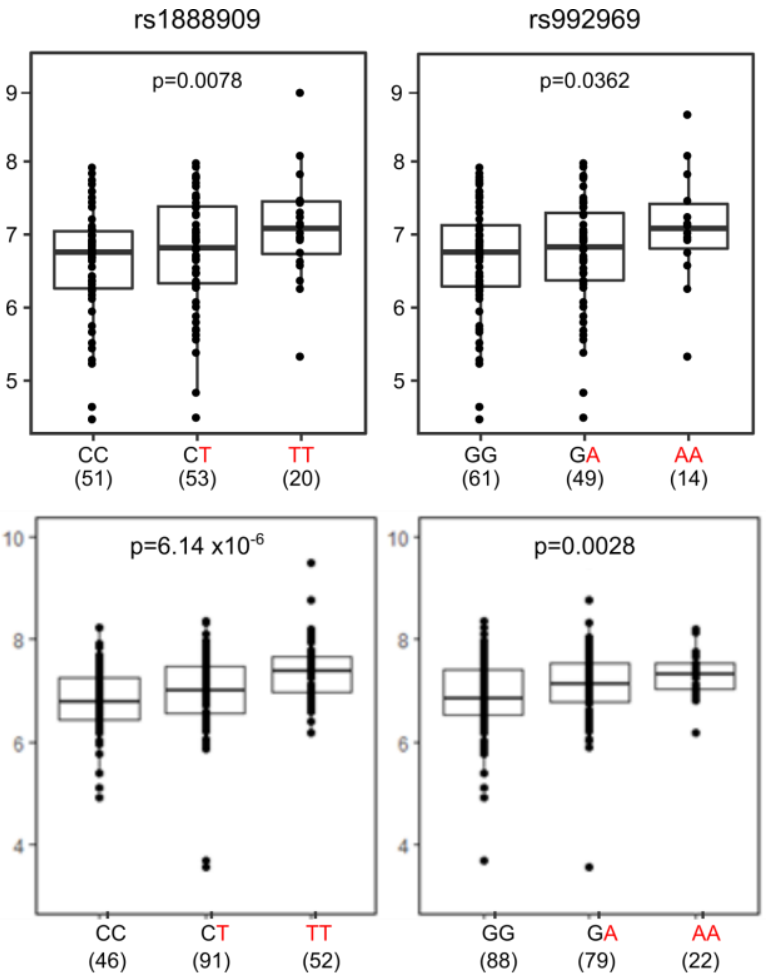

(22)
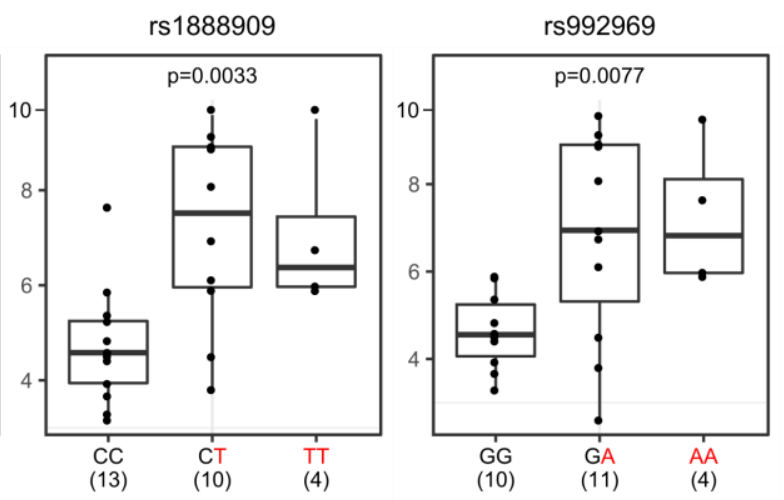

Figure 6. The rs1888909(T) and rs992969(A) alleles are associated with increased IL33 expression and IL-33 protein levels. Comparison of IL33 expression between genotypes for SNPs rs10975479, rs1888909 and rs992969 from a endobronchial brushings from 124 asthmatic and non-asthmatic adult subjects, mostly of European ancestry, and $\mathbf{b}$ nasal epithelial cells from 189 African American children from high risk asthma families. c Comparison of IL-33 cytokine levels between genotypes for SNPs rs10975479 ( $n=26)$, rs1888909 ( $n=27)$ and rs992969 ( $n=25)$ measured in plasma from Hutterite children (all European ancestry). The asthma-associated risk allele at each SNP is highlighted in red (x axis). The number of subjects per group is shown below the genotype. Boxes indicate the interquartile range, whiskers represent the $95 \%$ confidence intervals. Statistical significance was determined using an additive linear mode 


\section{Discussion}

We described an integrated pipeline to fine-map and functionally annotate the asthma-associated locus that includes the IL33 gene. We used the LD structure at this

462 locus across populations of different ethnicities combined with a Bayesian fine-mapping

463 tool to define a critical $20 \mathrm{~kb}$ genomic interval containing candidate causal SNPs for the

464 asthma association. Epigenetic signatures further reduced this region to $5 \mathrm{~kb}$, which we

465 demonstrated to have a crucial role in the development of chromatin loops creating

466 contacts between IL33 promoters and regulatory elements within the critical interval to

467 control IL33 expression. Associations between rs1888909 (T) allele copies and

468 increased IL33 mRNA and IL-33 protein levels further suggests that this regulatory

469 variant mediates the development of asthma among individuals carrying this risk allele.

The $5 \mathrm{~kb}$ region that we delineated and characterized in this study defies the

471 standard definition of regulatory elements. A loss of function assay showed that this

472 region is necessary for IL33 expression in vivo, suggestive of an enhancer function.

473 However, we failed to detect enhancer activity in reporter assays in cells where the

474 region displays chromatin markers associated with enhancers. Moreover, ENCODE

475 profiled the binding of hundreds of transcription factors across multiple cell types and

476 reports no binding of transcription activators, transcriptional co-factors, RNA Polll or

477 other factors usually associated with enhancers within this $5 \mathrm{~kb}$ region. Rather, the

478 region is bound by CTCF and cohesin in most cell lines assayed by ENCODE. We

479 showed that this region possesses enhancer blocking activity in vivo and in vitro,

480 reminiscent of insulator activities. Nevertheless, the region does not appear to behave

481 as a classically described insulator, as we fail to see a change in chromatin topology 
482 and long-range interactions in the locus secondary to the deletion of the $5 \mathrm{~kb}$ region in

483 the human BAC, which are typically seen when insulators are deleted ${ }^{30}$. While we

484 cannot ascribe a well-defined category for this regulatory element, we were able to

485 dissect its functional properties, to fine-map a variant that is likely causal to the

486 association with asthma and to identify the molecular effector binding to this regulatory

487 variant.

CTCF plays a critical role in chromatin loop formation and participates in demarcation of Topological Association Domains (TADs). Loop formation within TADs

490 facilitates contacts between specific genes and enhancers, allowing for appropriate 491 temporal and tissue-specific expression ${ }^{34}$. We observed that the $5 \mathrm{~kb}$ risk haplotype

492 carrying the asthma-associated allele possessed reduced enhancer blocking function in 493 both in vitro and in vivo assays, and identified a smaller $1 \mathrm{~kb}$ fragment which maintained

494 enhancer blocking activity. Interestingly, the loss of a 400bp region within this $1 \mathrm{~kb}$

495 fragment, harboring 2 asthma-associated SNPs, led to a significant loss of function, 496 demonstrating a requirement for factors binding in that region for its proper regulatory 497 activity.

We identified OCT-1 as a transcription factor that binds differentially to the risk

499 allele of rs1888909, thus implicating OCT-1 in the function(s) of this $5 \mathrm{~kb}$ region. OCT-1

500 can regulate gene expression both positively and negatively. It has been shown to bind

501 enhancers and regulatory regions upstream of multiple cytokines, including IL-3 $3^{35}$, IL-

$50212 \mathrm{p} 40^{36}, \mathrm{IL}-13^{37}, \mathrm{IL}-4^{38}$ and IL-1739. The mechanisms by which OCT-1 functions are

503 strikingly diverse, and there are numerous studies reporting the coordinated activity of

504 CTCF and OCT-1. For example, at the IL17 gene cluster on chromosome 3, OCT-1 and 
505 CTCF facilitate long-range associations with the IL-2 locus in naïve T cells. In a parallel

506 fashion, it is possible that OCT-1 coordinates with CTCF to direct IL33 expression at the

507 locus through creation of appropriate chromatin interactions between enhancers and the

508 IL33 promoter. The distinct binding complexes formed on the risk and non-risk alleles at

509 the rs1888909 genomic region may be responsible for alterations in these interactions,

510 which in turn may lead to altered IL33 expression levels.

511 While our data support a role of rs1888909 in the regulation of IL33 expression

512 and its primary role with asthma risk, our fine mapping results identified two other

513 potential causative sets of SNPs associated with asthma at the IL33 locus, upstream

514 and downstream to the region that we studied (Fig1b). This suggests that other variants

515 in this locus may have independent effects that alter the function of other regulatory

516 elements and potentially controls expression of IL33 or other genes. Future studies will

517 be needed to further dissect the genetic architecture at this complex locus and address

518 this hypothesis.

In summary, we identified a small $5 \mathrm{~kb}$ noncoding interval which is integral to

$520 \quad$ IL33 expression in several cell types. We propose a model in which CTCF mediates

521 interaction of the $5 \mathrm{~kb}$ region to the IL33 promoters through the formation of chromatin

522 loops. The interplay between the distinct regulatory elements in the locus promotes

523 spatial and temporal-specific regulation which is affected by OCT-1 binding to

524 rs1888909. These results together with the increased IL33 expression observed in

525 humans with the risk allele offer a plausible mechanistic explanation for the association

526 of these variants and asthma risk. A deeper characterization of these mechanisms will

527 not only enhance our understanding of IL33 regulation but may also open new 
bioRxiv preprint doi: https://doi.org/10.1101/2020.09.09.290098; this version posted September 11, 2020. The copyright holder for this preprint (which was not certified by peer review) is the author/funder. All rights reserved. No reuse allowed without permission.

528 strategies in directing future studies, as well as other common diseases in which

529 dysregulation of IL-33 plays a role. 


\section{$530 \quad$ Materials and Methods.}

\section{Experimental animals}

532 Generation of $\mathrm{hl} L 33^{\mathrm{Crm}} \mathrm{BAC}$ and $\mathrm{h} / \mathrm{L} 33^{\mathrm{Crm}} 5 \mathrm{Kdel}$ transgenic mice was performed by the

533 University of Chicago Transgenic Core Facility. Modified DNA was diluted to a

534 concentration of $2 \mathrm{ng} / \mu \mathrm{l}$ and used for pronuclear injections of CD1 embryos in

535 accordance with standard protocols approved by the University of Chicago.

\section{Red/ET BAC modification}

538 BAC RP11725F15, obtained from the BACPAC Resource Center (Oakland, CA), was

539 modified in vitro using the RED/ET recombination kit (Gene Bridges, Heidelberg

540 Germany) according to the manufacturer's instructions.

541 Crimson- kanamycin cassette was PCR amplified from vector pE2-Crimson-N1 vector.

542 The reporter cassette was inserted in frame to the ATG of the human IL33 gene, to

543 replace the second exon of the gene, while maintaining boundaries and flanking regions

544 fully intact. Primers containing 50-bp BAC-homology arms used for generation of the

545 recombination cassette were as follow: IL33_CrimsonKanF:

546 TTGAGACAAATGAACTAATATTATATTTTAATCCAACAGAATACTGAAAAATGGATAG

547 CACTGAGAACGTCA; IL33_CrimsonKanR,

548 GCGTAAAACATTCAGAGATAACTTAAGTCCTTACTTCCCAGCTTGAAACATCAGAAG

549 AACTCGTCAAGAAGG. Successful recombinants were screened for homologous

550 insertion using the following external/internal primers IL33F-Crm5',

551 AGCCACAGTTGTTTCCGTTT; IL33R-Crm5', TTGAGGTAGTCGGGGATGTC and IL- 
554 The asthma- associated $5 \mathrm{~kb}$ interval (chr9: 6,194,500-6,199,500; hg19) was deleted

555 using RED/ET recombination kit (Gene Bridges). The $5 \mathrm{~kb}$ region of interest was

556 replaced by the ampicillin gene using the following primers containing $50 \mathrm{pb}$ homology

557 arms flanking the BAC region to be deleted. IL33InsDEL_F,

558 GCACACCTGTAAGTCTCTGCATTTTGCCACTTATACAACTTCATCTTTGAGTGGCAC

559 TTTTCGGGGAAATG; IL33InsDEL_R,

560 AAACTTACATCAAATAAAATCTCAACACAGAATTCATACATGTCAACATACTCGAGG

561 CTAGCTCTAGAAGTC. All correctly modified Bacs were verified by fingerprinting and

562 sequencing. BAC DNA was extracted using the Nucleobond PC20 kit (Macherey-Nagel)

563 and diluted for pronuclear injection. BAC copy number was determined as previously

564 described 40

\section{Quantitative RT-PCR}

567 RNA from flash frozen tissues were extracted from control and insulator deleted mice

568 using TRI-reagent (Sigma). cDNA was generated using Super Script II (Thermo). IL33

569 primers (5'GCCAAGCTGCAAGTGACCAA 3'; 5'GCCTTGGAGCCGTAGAAGAA 3') and

570 HPRT housekeeping gene primes were used to quantify IL33 mRNA expression levels.

571 RNA samples with no reverse transcriptase added were used to test for genomic

572 contamination. 
577 Mouse lymph nodes were embedded into blocks with Optimal Cutting Temperature 578 compound (OCT 4583) and stored at $-80^{\circ} \mathrm{C}$. Frozen tissues were sliced into sections

$5795 \mu \mathrm{m}$ thick and dried onto slides overnight. Sections were fixed, permeabilized,

580 quenched and blocked. Tissues were immunostained with the primary antibodies rat

581 anti-mouse CD31:Biotin (clone 390, Biolegend, San Diego, CA, USA) and Living Colors

582 DsRed Polyclonal Antibody (rabbit anti-E2-Crimson, Clontech [now Takara Bio USA],

583 Mountain View, CA, USA). Sections were washed and stained with the secondary

584 antibodies Streptavidin:Alexa Fluor 488 (Biolegend, San Diego, CA, USA) and Goat

585 anti-Rabbit IgG:Alexa Fluor 633 (Life Technologies, Thermo Fisher, Waltham, MA,

586 USA), along with the nucleic acid stain Hoechst 33342 (Life Technologies, Thermo

587 Fisher, Waltham, MA, USA). Coverslips were set with ProLong Diamond Antifade

588 Mountant (Life Technologies, ThermoFisher, Waltham, MA, USA). Imaging was

589 performed at the University of Chicago Integrated Light Microscopy Facility. Images

590 were captured with a Leica TCS SP2 laser scanning confocal microscope (Leica

591 Microsystems, Inc., Buffalo Grove, IL, USA) using a 63x/1.4 UV oil immersion objective

592 and LAS_AF acquisition software (Leica Microsystems, Inc., Buffalo Grove, IL, USA).

593 Further processing was completed using ImageJ software (National Institutes of Health, 594 Bethesda, MD, USA).

596 Promoter capture in situ Hi-C

597 Promoter capture $\mathrm{Hi}-\mathrm{C}(\mathrm{PCHiC})$ data for human lymphoblast cells (LCL) was obtained 598 from NCBI GEO (accession number: GSE79718), mapped to human genome assembly 
599 hg19, and processed as previously described ${ }^{41,42}$. Interactions were visualized using then

600 WashU Epigenome Browser.

\section{Circularized Chromosome Conformation Capture sequencing (4C-seq)}

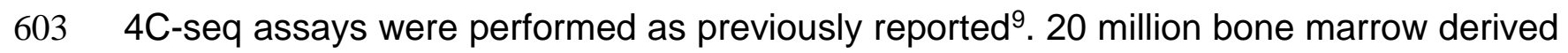
604 dendritic cells were obtained from control and insulator deleted mice. Immediately before 605 4C-seq library preparation, cells were cross-linked with 1\% fresh formaldehyde for 15 606 minutes and treated with lysis buffer $(10 \mathrm{mM}$ Tris- $\mathrm{HCl} \mathrm{pH}$ 8, $10 \mathrm{mM} \mathrm{NaCl}, 0.3 \%$ IGEPAL 607 CA-630 (Sigma-Aldrich), 1X complete protease inhibitor cocktail (Roche). Nuclei were 608 digested with Csp6-I endonuclease (Life Technologies, ThermoFisher, Waltham, MA) 609 and ligated with T4 DNA ligase (Promega, Madison, WI) for 12 hours at $4^{\circ} \mathrm{C}$, followed by 610 reverse crosslinking at $65^{\circ} \mathrm{C}$ for 12 hours with proteinase K. Subsequently, Nlalll 611 endonuclease (New England Biolabs) was used in a second round of digestion, and the 612 DNA was ligated again. Specific primers were designed near the $5 \mathrm{~kb}$ region of interest.

613 Viewpoint fragment ends (fragends) coordinates was chr9:6,186,164-6,186,468. Primers 614 used for 4C-Seq experiments are as follow: 4CmIL33IL33Enh_nonread, 5' 615 CAAGCAGAAGACGGCATACGAACAACTTCACTCAGAGGCATG 3' and 616 4CmIL33IL33Enh_Read 5' 617 AATGATACGGCGACCACCGAACACTCTTTCCCTACACGACGCTCTTCCGATCTGAG 618 ATGGCGCCACTGTAC 3' 


\section{Constructs preparation}

623 PCR fragments of genomic DNA from a risk and non-risk cell line were cloned into the

624 pDONR vector (Life Technologies, ThermoFisher, Waltham, MA) and sequenced by the

625 University of Chicago DNA Sequencing and Genotyping facility. DNA was subcloned

626 into the enhancer barrier vector ${ }^{28}$ (gift from Dr. Laura Elnitski, NHGRI) or pGL4.23 for

627 enhancer assays (Promega, Madison, WI). Constructs were prepared using the Plasmid

628 MidiPrep Kit (Qiagen, Hilden, Germany) and re-sequenced to confirm genotype.

$630 \quad$ Luciferase assay

631 K562 erythroleukemic were transfected using TransIT-2020 Transfection Reagent

632 (Mirus Bio, Madison, WI). Briefly, $10^{5}$ cells per well were plated 24 hours prior to

633 transfection. The transfection mix included $0.5 \mu \mathrm{g}$ of barrier or enhancer plasmid, $10 \mathrm{ng}$

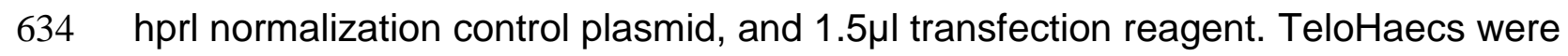

635 transfected using JetPRIME transfection reagent (Polyplus). 20,000 cells per well (24

636 well plate) were plated and transfect with $250 \mathrm{ng}$ of test plasmid DNA and $25 \mathrm{ng}$ of hprl

637 normalization control plasmid. After 48 hours cells were harvested and assayed for

638 luciferase with a 20/20n luminometer (Turner Biosystems, now Fisher Scientific,

639 Hampton, NH) using the Dual Luciferase Reporter Assay System (Promega, Madison,

640 WI). Firefly luciferase was normalized to the Renilla luciferase. A minimum of three

641 independent transfections using 3 different DNA preparations were assayed. 


\section{Zebrafish transgenesis}

645 The Tol2 vector contains a strong midbrain enhancer, a Gateway entry site and the

646 cardiac actin promoter controlling the expression of EGFP, and was developed to

647 screen for insulator activity ${ }^{29}$. Each candidate sequence was recombined between the

648 midbrain enhancer and the cardiac actin promoter. As a reference, the empty backbone

649 was used (INS-zero). One cell-stage embryos were injected with 3-5 nL of a solution

650 containing $25 \mathrm{nM}$ of each construct plus $25 \mathrm{nM}$ of Tol2 mRNA. Embryos where then

651 incubated at $28^{\circ} \mathrm{C}$ and EGFP expression was evaluated $24 \mathrm{hpf}$. The midbrain/somites

652 EGFP intensity ratio was quantified using ImageJ freeware and was directly proportional

653 to the enhancer-blocking capacity. As a positive control, the chicken beta-globin

654 insulator 5HS4 was used. Each experiment was repeated independently and double-

655 blinded to the operators.

\section{EMSA}

658 Nuclear extracts were prepared from K562 cells using NE-PER Nuclear and

659 Cytoplasmic Extraction Kit (Thermo Fisher, Waltham, MA) supplemented with HALT

660 Protease Inhibitor Cocktail and PMSF (Thermo Fisher, Waltham, MA). Protein

661 concentrations were determined with the BCA kit (Thermo Fisher, Waltham,MA).

662 Oligonucleotides to be used as probes were synthesized with a 5'IRDye 700

663 modification (IDT). Binding reactions were performed using the Odyssey EMSA kit (LI-

664 COR Biosciences, Lincoln, NE) and contained 5 $\mathrm{gg}$ nuclear extract, 2.5nM labeled

665 probe, and when noted, 100x excess unlabeled oligonucleotide. For supershift assays,

$6662 \mu \mathrm{g}$ Oct-1 antibody (Santa Cruz Biotechnology, Dallas, TX, sc-232) was added and 
667 incubated for another 20 minutes. Reaction mixtures were run on a $4 \%$ nondenaturing

668 polyacrylamide gel and analyzed with the LI-COR Odyssey Imaging System.

670 Oligonucleotide/Probe sequences:

671 479G:909 TCTGATGCAGAACAGCAATGTGTTTTCCAIGTGCACTTGGTC

672 479G:909 CCTGATGCAGAACAGCAATGTGTTTTCCACGGTGCACTTGGTC

673 479A:909 TCTGATGCAGAACAACAATGTGTTTTCCAIGTGCACTTGGTC

674 479A:909 CCTGATGCAGAACAㅁAATGTGTTTTCCAㅡGTGCACTTGGTC

675 Consensus Oct1 TGTCGAATGCAAATCACTAGAA

676 Mutant Oct1 TGTCGAATGCAAGCCACTAGAA.

678 Mass Spectrophotometry and protein identification

679 The gel sections to be analyzed from the EMSA gel were excised, washed and

680 destained using $100 \mathrm{mM} \mathrm{NH} 4 \mathrm{HCO} 3$ in $50 \%$ acetonitrile (ACN). Sections then underwent

681 reduction, alkylation, and trypsinization. Peptides were extracted with 5\% formic acid,

682 followed by $75 \%$ ACN in formic acid, and cleaned up with C-18 spin columns (Pierce).

683 The samples were analyzed via electrospray tandem spectrometry (LC-MS/MS) on a

684 Thermo Q-Exactive Orbitrap mass spectrometer at the Proteomics Core at Mayo Clinic,

685 Rochester, Minnesota. Tandem mass spectra were extracted and then analyzed by

686 Mascot and X!Tandem algorithms. Scaffold v4.8.4 (Proteome Software Inc.,

687 Portland,OR) was used to validate the protein identifications. Peptide identifications 
688 were accepted if they could be established at greater than $98 \%$ probability to achieve an

689 FDR less than $1.0 \%$.

690

691 Candidate Transcription factor analysis

692 Panther pathway analysis database ${ }^{43}$ was used to filter for DNA-binding proteins.

693 Jaspar (http://jaspar.genereg.net) and Alggen-promo (http://alggen.Isi.upc.es)

694 databases were used to screen these factors for known DNA binding sites. We

695 downloaded binding data for random octamers from UniProbe

696 (http://thebrain.bwh.harvard.edu/pbms/webworks pub dev/downloads.php) and

697 identified all transcription factors that bound at least one octamer containing a risk or

698 non-risk allele.

699

700 Chromatin Immunoprecipitation

701 ChIP assays were performed using the Millipore ChIP assay kit and protocol. $2 \times 10^{6}$

$702 \mathrm{H} 292$ cells (heterozygous for the risk allele) were fixed with $1 \%$ formaldehyde.

703 Chromatin was sonicated using a diagenode BioRuptor. Lysates were incubated

704 overnight with 5ug of Oct-1 (Santa Cruz, sc-232x) or an IgG control (Santa Cruz, sc-

705 2027). Following the washes, elution of chromatin complexes and reversal of crosslinks,

706 DNA was recovered using Qiagen pcr purification kit. Input DNA was also processed.

707 QPCR was performed using the primers: F: 5'GCCTCTGGTCTCAGTGGATA3' and

708 R: 5'CTGCTCATAGGAGACACAGTAAAG3'. 


\section{Gene expression and genotype studies}

711 RNA-seq data from airway epithelial cells were available from two sources. The first

712 was from bronchial epithelial cells sampled from adults (44 European American, 48

713 African American), with and without asthma (76 cases, 60 controls), who participated in

714 a study of asthma in Chicago. Procedures for bronchoscopy, cell and RNA processing,

715 and genotyping have been previously described ${ }^{41}$. For this study, normalized gene

716 expression counts were adjusted for age, sex, current smoking status, sequencing pool,

717 the first three ancestry PCs. Linear regression considering additive genotype effects on

718 gene expression was performed using limma in R (v3.3.3). $P$-values were adjusted for 3

719 tests; $P<0.016$ was considered significant. All subjects provided written informed

720 consent; these studies were approved by University Chicago Institutional Review Board.

The second source was nasal epithelial cells sampled from 246 African American

722 children (125 cases, 121 controls) from a birth cohort of children at high risk for

723 asthma ${ }^{44}$. Procedures for nasal brushing and cell processing followed standard

724 procedures ${ }^{45}$. Genotypes were determined using Illumina Multi-Ethnic Genotyping Array

725 (MEGA), and processed using standard $\mathrm{QC}^{41}$. To test for association between

726 genotypes for three SNPs and IL33 transcript levels, we used an additive effects linear

727 model, including as covariates sex, study site, batch id, epithelial cell proportion and 12

728 latent factors in the epithelial cell studies. Latent factors were included to correct for

729 unwanted variation ${ }^{46} . P$-values were adjusted for 3 tests; $P<0.016$ was considered

730 significant. Parents of all children provided written informed consent, and children

731 provided written assent, for genetic studies; these studies were approved by University

732 Chicago Institutional Review Board. 


\section{IL-33 cytokine and genotype studies}

734 Blood for cytokine studies was drawn from 30 Hutterite children during trips to South

735 Dakota, as previously described ${ }^{47}$. Written consent was obtained from the parents and

736 written assent was obtained from the children. The study was approved by the

737 institutional review boards at the University of Chicago. Briefly, The Milliplex Map

738 Human TH17 Magnetic Bead Panel (Millipore, Burlington, MA) was used to measure IL-

73933 levels in thawed supernatants at the University of Chicago Immunology Core facility

740 using standard protocols, as previously described. ${ }^{48}$

Associations between genotypes at rs1888909, rs992969 and rs10975479 and

742 normalized IL-33 cytokine abundance were tested using a linear model, assuming

743 additive effects. Prior to analysis, voom-transformed gene expression counts were

744 adjusted for age, sex, current smoking status, sequencing pool and the first three

745 ancestry PCs using the function removeBatchEffect from the $\mathrm{R}$ package limma ${ }^{49}$. Linear

746 regression between the genotypes and IL-33 levels was performed with the FastQTL

747 software package ${ }^{50}$. One subject had poor IL-33 cytokine data and was removed. Of the

748 remaining subjects, 27 subjects for rs 10975479 and rs 1888909 and 25 subjects for

749 rs992969 had good quality genotype calls and were retained. Linear regression

750 between the genotypes and IL-33 cytokine levels was performed using limma, adjusting

751 for sex and age. Genotypes were obtained using PRIMAL ${ }^{51}$, an in-house pedigree-

752 based imputation tool that imputes variants from whole genome sequences from 98

753 Hutterite individuals to $>1600$ individuals who were genotyped with an Affymetrix

754 genotyping array. 


\section{Acknowledgements}

757 We thank Don Wolfgeher, from the University of Chicago's Proteomics Core Facility, for

758 assistance with sample preparation and analysis of the mass spectrophotemetry; Dr.

759 Laura Elnitski (NHGRI/NIH, Bethesda,MD), for generously supplying the enhancer

760 barrier plasmids. Dr. Benjamin Glick (University of Chicago) for supplying the E2-

761 Crimson reporter vector.

762

763

\section{Disclosures}

765 The authors have no financial conflict of interest. 


\section{REFERENCES}

768 1. Polderman, T.J. et al. Meta-analysis of the heritability of human traits based on fifty years of twin studies. Nature genetics 47, 702-709 (2015).

2. Pividori, M., Schoettler, N., Nicolae, D.L., Ober, C. \& Im, H.K. Shared and distinct genetic risk factors for childhood-onset and adult-onset asthma: genome-wide and transcriptome-wide

773 studies. Lancet Respir Med 7, 509-522 (2019).

3. Schmitz, J. et al. IL-33, an interleukin-1-like cytokine that signals via the IL-1 receptor-related protein ST2 and induces T helper type 2-associated cytokines. Immunity 23, 479-490 (2005).

4. Prefontaine, D. et al. Increased expression of IL-33 in severe asthma: evidence of expression by airway smooth muscle cells. J Immunol 183, 5094-5103 (2009).

5. Kearley, J., Buckland, K.F., Mathie, S.A. \& Lloyd, C.M. Resolution of allergic inflammation and airway hyperreactivity is dependent upon disruption of the T1/ST2-IL-33 pathway. Am J Respir Crit Care Med 179, 772-781 (2009).

6. Savinko, T. et al. IL-33 and ST2 in atopic dermatitis: expression profiles and modulation by triggering factors. J Invest Dermatol 132, 1392-1400 (2012).

7. Kamekura, R. et al. The role of IL-33 and its receptor ST2 in human nasal epithelium with allergic rhinitis. Clin Exp Allergy 42, 218-228 (2012).

8. Kottyan, L.C. et al. Genome-wide association analysis of eosinophilic esophagitis provides insight into the tissue specificity of this allergic disease. Nature genetics 46, 895-900 (2014).

9. Smemo, S. et al. Obesity-associated variants within FTO form long-range functional connections with IRX3. Nature 507, 371-375 (2014).

10. van den Boogaard, M. et al. A common genetic variant within SCN10A modulates cardiac SCN5A expression. J Clin Invest 124, 1844-1852 (2014).

11. Rusu, V. et al. Type 2 Diabetes Variants Disrupt Function of SLC16A11 through Two Distinct Mechanisms. Cell 170, 199-212 e120 (2017).

12. Small, K.S. et al. Regulatory variants at KLF14 influence type 2 diabetes risk via a female-specific effect on adipocyte size and body composition. Nature genetics 50, 572-580 (2018).

13. Wasserman, N.F., Aneas, I. \& Nobrega, M.A. An 8q24 gene desert variant associated with prostate cancer risk confers differential in vivo activity to a MYC enhancer. Genome Res 20, 1191-1197 (2010).

14. Gallagher, P.G. et al. Mutation of a barrier insulator in the human ankyrin-1 gene is associated with hereditary spherocytosis. J Clin Invest 120, 4453-4465 (2010). 
15. Moffatt, M.F. et al. A large-scale, consortium-based genomewide association study of asthma. $N$ Engl J Med 363, 1211-1221 (2010).

16. Torgerson, D.G. et al. Meta-analysis of genome-wide association studies of asthma in ethnically

17. Bonnelykke, K. et al. A genome-wide association study identifies CDHR3 as a susceptibility locus for early childhood asthma with severe exacerbations. Nature genetics 46, 51-55 (2014).

18. Demenais, F. et al. Multiancestry association study identifies new asthma risk loci that colocalize with immune-cell enhancer marks. Nat Genet 50, 42-53 (2018).

19. Daya, M. et al. Association study in African-admixed populations across the Americas recapitulates asthma risk loci in non-African populations. Nature communications 10,880 (2019).

20. Ferreira, M.A.R. et al. Genetic Architectures of Childhood- and Adult-Onset Asthma Are Partly Distinct. Am J Hum Genet 104, 665-684 (2019).

21. Zhu, X. \& Stephens, M. Bayesian Large-Scale Multiple Regression with Summary Statistics from Genome-Wide Association Studies. Ann Appl Stat 11, 1561-1592 (2017).

22. Bycroft, C. et al. The UK Biobank resource with deep phenotyping and genomic data. Nature 562, 203-209 (2018).

23. Roadmap Epigenomics, C. et al. Integrative analysis of 111 reference human epigenomes. Nature 518, 317-330 (2015).

24. Consortium, E.P. A user's guide to the encyclopedia of DNA elements (ENCODE). PLoS Biol 9, e1001046 (2011).

25. Kim, S., Yu, N.K. \& Kaang, B.K. CTCF as a multifunctional protein in genome regulation and gene expression. Exp Mol Med 47, e166 (2015).

26. Chen, D. \& Lei, E.P. Function and regulation of chromatin insulators in dynamic genome organization. Curr Opin Cell Biol 58, 61-68 (2019).

27. Pichery, M. et al. Endogenous IL-33 Is Highly Expressed in Mouse Epithelial Barrier Tissues, Lymphoid Organs, Brain, Embryos, and Inflamed Tissues: In Situ Analysis Using a Novel II-33-LacZ Gene Trap Reporter Strain. J Immunol 188, 3488-3495 (2012).

28. Petrykowska, H.M., Vockley, C.M. \& Elnitski, L. Detection and characterization of silencers and enhancer-blockers in the greater CFTR locus. Genome Res 18, 1238-1246 (2008).

29. Bessa, J. et al. Zebrafish enhancer detection (ZED) vector: a new tool to facilitate transgenesis and the functional analysis of cis-regulatory regions in zebrafish. Dev Dyn 238, 2409-2417 (2009). 
30. Flavahan, W.A. et al. Altered chromosomal topology drives oncogenic programs in SDH-deficient

862

863

864

865

866

867

868 GISTs. Nature 575, 229-233 (2019).

31. Hume, M.A., Barrera, L.A., Gisselbrecht, S.S. \& Bulyk, M.L. UniPROBE, update 2015: new tools and content for the online database of protein-binding microarray data on protein-DNA interactions. Nucleic Acids Res 43, D117-122 (2015).

32. Li, X. et al. eQTL of bronchial epithelial cells and bronchial alveolar lavage deciphers GWASidentified asthma genes. Allergy (2015).

33. Stein, M.M. et al. Innate Immunity and Asthma Risk in Amish and Hutterite Farm Children. $N$ Engl J Med 375, 411-421 (2016).

34. West, A.G., Gaszner, M. \& Felsenfeld, G. Insulators: many functions, many mechanisms. Genes Dev 16, 271-288 (2002).

35. Duncliffe, K.N., Bert, A.G., Vadas, M.A. \& Cockerill, P.N. A T cell-specific enhancer in the interleukin-3 locus is activated cooperatively by Oct and NFAT elements within a DNase Ihypersensitive site. Immunity 6, 175-185 (1997).

36. Zhou, L. et al. An inducible enhancer required for $1112 \mathrm{~b}$ promoter activity in an insulated chromatin environment. Mol Cell Biol 27, 2698-2712 (2007).

37. Kiesler, P., Shakya, A., Tantin, D. \& Vercelli, D. An allergy-associated polymorphism in a novel regulatory element enhances IL13 expression. Hum Mol Genet 18, 4513-4520 (2009).

38. Kim, K., Kim, N. \& Lee, G.R. Transcription Factors Oct-1 and GATA-3 Cooperatively Regulate Th2 Cytokine Gene Expression via the RHS5 within the Th2 Locus Control Region. PLoS One 11, e0148576 (2016).

39. Kim, L.K. et al. Oct-1 regulates IL-17 expression by directing interchromosomal associations in conjunction with CTCF in T cells. Mol Cell 54, 56-66 (2014).

40. Chandler, K.J. et al. Relevance of BAC transgene copy number in mice: transgene copy number variation across multiple transgenic lines and correlations with transgene integrity and expression. Mamm Genome 18, 693-708 (2007).

41. Nicodemus-Johnson, J. et al. DNA methylation in lung cells is associated with asthma endotypes and genetic risk. JCl Insight 1, e90151 (2016).

42. Nicodemus-Johnson, J. et al. Genome-Wide Methylation Study Identifies an IL-13-induced Epigenetic Signature in Asthmatic Airways. Am J Respir Crit Care Med 193, 376-385 (2016).

43. Mi, H., Muruganujan, A., Ebert, D., Huang, X. \& Thomas, P.D. PANTHER version 14: more genomes, a new PANTHER GO-slim and improvements in enrichment analysis tools. Nucleic Acids Res 47, D419-D426 (2019). 
908

909

910

911

912

913

914

915

916

917

918

919

920

921

922

923

924

925

926

927

928

929

930

931

932

933

934
44. Gern, J.E. et al. The Urban Environment and Childhood Asthma (URECA) birth cohort study: design, methods, and study population. BMC Pulm Med 9, 17 (2009).

45. Poole, A. et al. Dissecting childhood asthma with nasal transcriptomics distinguishes subphenotypes of disease. J Allergy Clin Immunol 133, 670-678 e612 (2014).

46. McKennan, C. \& Nicolae, D.L. Accounting for unobserved covariates with varying degree of estimability in high dimensional experimental data. Biometrika 106, 823-840 (2019).

47. Stein, M.M. et al. Innate Immunity and Asthma Risk in Amish and Hutterite Farm Children. $N$ Engl J Med 375, 411-421 (2016).

48. Stein, M.M., Hrusch, C.L., Sperling, A.I. \& Ober, C. Effects of an FcgammaRIIA polymorphism on leukocyte gene expression and cytokine responses to anti-CD3 and anti-CD28 antibodies. Genes Immun (2018).

49. Ritchie, M.E. et al. limma powers differential expression analyses for RNA-sequencing and microarray studies. Nucleic Acids Res 43, e47 (2015).

50. Ongen, H., Buil, A., Brown, A.A., Dermitzakis, E.T. \& Delaneau, O. Fast and efficient QTL mapper for thousands of molecular phenotypes. Bioinformatics 32, 1479-1485 (2016).

51. Livne, O.E. et al. PRIMAL: Fast and Accurate Pedigree-based Imputation from Sequence Data in a Founder Population. PLoS Comput Biol 11, e1004139 (2015). 